\title{
PARTIAL MIELNIK SPACES AND CHARACTERIZATION OF UNIFORMLY CONVEX SPACES
}

\author{
A. R. BLASS AND C. V. STANOJEVIC
}

\begin{abstract}
We characterize uniform convexity of normed linear spaces in terms of a functional inequality generalizing Clarkson's inequality for $L_{p}$ spaces. This inequality can be interpreted as saying that the unit sphere of the space carries a structure slightly weaker than a probability space in the sense of Mielnik. From this point of view, our result is analogous to an earlier characterization of inner product spaces. We also investigate briefly the abstract concept of partial probability space suggested by the main result.
\end{abstract}

1. Introduction. The classical approach to the foundations of quantum theory, pioneered by Birkhoff and von Neumann [1], sought axioms for quantum logic all of whose realizations could be represented by orthogonal projection operators in suitable Hilbert spaces. B. Mielnik [4] objected that this program would not fully justify the use of Hilbert spaces in quantum theory, for that theory deals not only with the yes-no measurements considered in quantum logic, but also with statistical predictions. Accordingly, he introduced the following concept of probability space, and showed that not all of its realizations could be obtained from Hilbert spaces in the usual quantumtheoretic manner.

A Mielnik space $(S, p)$ consists of a nonempty set $S$ together with a function $p$ from $S \times S$ into the closed interval $[0,1]$ of the real line satisfying the following three axioms.

(A) $p(a, b)=1$ if and only if $a=b$.

(B) $p(a, b)=p(b, a)$. To state the last axiom concisely, we first define two members $a$ and $b$ of $S$ to be orthogonal if $p(a, b)=0$. A maximal set of pairwise orthogonal elements of $S$ is called a basis of $S$. The existence of bases is an easy consequence of Zorn's lemma.

(C) For any $a \in S$ and any basis $B$ of $S, \sum_{b \in B} p(a, b)=1$, where the (possibly infinite or even uncountable sum) is interpreted, as usual, as the supremum of all its finite partial sums.

The elements of $S$ are to be viewed as possible states of a physical system, and $p(a, b)$ as the probability that a system is found to be in state $b$ after being known to be in state $a$. In the usual quantum-mechanical formalism, $S$ is the set of unit vectors of a Hilbert space (modulo identification of a vector $a$ with all its scalar multiples $\lambda a,|\lambda|=1)$ and $p(a, b)=|\langle a, b\rangle|^{2}$. A basis for an $S$ of this sort is just an orthonormal basis for the Hilbert space. Mielnik [4] showed

Received by the editors July 18, 1974 and, in revised form, September 1, 1974.

AMS (MOS) subject classifications (1970). Primary 46B99, 81A12.

Key words and phrases. Uniformly convex spaces, Mielnik probability spaces. 
that, in any probability space $(S, p)$, all bases have the same cardinality, called the dimension of $(S, p)$. Thus, the dimension of the Mielnik spaces used in quantum mechanics is the same as the dimension of the associated Hilbert space.

There is another way to associate a Mielnik space with any given inner product space $N$. Let $S$ be the unit sphere of $N$ (without identifying vectors that differ by a phase factor), and let

$$
p(a, b)=\frac{1}{4}\|a+b\|^{2} .
$$

Although only the norm of $N$, not the inner product, appears explicitly in the definition of $(S, p)$, the fact that the norm is associated to an inner product is used in verifying that $(S, p)$ satisfies Mielnik's Axiom (C) (the other two axioms being immediate). Indeed, the only vector orthogonal to $a$ in $(S, p)$ is $-a$, so (C) reads

$$
\frac{1}{4}\|a+b\|^{2}+\frac{1}{4}\|a-b\|^{2}=1,
$$

which follows from the parallelogram law. Notice that $(S, p)$ has dimension 2; among these spaces are Mielnik's examples of Mielnik spaces not obtainable from Hilbert spaces in the usual quantum-mechanical way.

In [5] it was shown that formula (1) defined a Mielnik space structure on the unit sphere of a normed real linear space $N$ if and only if $N$ is an inner product space. In [6] this result was generalized to show that if $S$ is the unit sphere of a normed real linear space and if $(S, p)$ is a Mielnik space of dimension 2 in which $p(a, b)$ is any reasonable function of $\|a+b\|$, then $N$ is an inner product space. To state this result, and our later results, precisely, we introduce, as in [6], the class

$$
\begin{aligned}
\mathscr{F}=\{f \mid f:[0,2] \rightarrow[0,1], f \text { continuous and } \\
\text { strictly increasing, } f(0)=0, f(2)=1\} .
\end{aligned}
$$

Then Theorem 3.1 of [6] asserts that a normed linear space $N$ is an inner product space if and only if its unit sphere $S$, equipped with some $p$ of the form $p(a, b)=f(\|a+b\|), f \in \mathscr{F}$, is a Mielnik probability space (necessarily of dimension 2).

In this paper, we shall introduce the notion of a partial Mielnik space and use it to obtain a characterization of uniformly convex spaces analogous to the characterization of inner product spaces just quoted. We are not concerned with the axiomatic foundations of quantum theories, but rather with some important mathematical implications of Mielnik's work [4].

2. Partial Mielnik spaces. A partial Mielnik space is a pair $(S, p)$, where $S$ is a nonempty set and $p$ maps $S \times S$ into [0,1] in such a way that Axioms (A) and (B) for Mielnik spaces and the following weakened form of Axiom (C) hold.

(C*) For any $a \in S$ and any basis $B$ of $S, \sum_{b \in B} p(a, b) \leqslant 1$. Partial Mielnik spaces abound, for any nonempty subset of a Mielnik space is a partial Mielnik space. To see this, simply observe that a basis for such a subset is a subset of a basis for the whole Mielnik space. It is also easy to construct 
partial Mielnik spaces which are not subspaces of any Mielnik space. We shall consider later the problem of finding conditions under which a partial Mielnik space can be embedded in a Mielnik space.

Although there is no immediate physical interpretation of $\left(C^{*}\right)$, it is related to the behavior of quantum-mechanical transition probabilities between states of an unstable system. If we let $S$ consist of the states of such a system, say a neutron, and if we let $p(a, b)$ be the probability that a neutron known to be in state $a$ at a certain time is found to be in state $b$ ten minutes later, then Axiom (C) is not satisfied but Axiom $\left(C^{*}\right)$ is, because there is a nonzero probability that the neutron will, during the ten minute interval, decay into a proton, an electron, and an antineutrino. Notice the relation between this observation and the remarks in the preceding paragraph: Axiom (C) would hold if we added to $S$ all the states of the systems into which the neutron can decay. Notice also that our $(S, p)$ fails to be a partial Mielnik space because the "if" part of Axiom (A) is false (and Axiom (B) expresses the rather strong assumption of time-reversal invariance). It would perhaps be reasonable to weaken Mielnik's axioms further by omitting the "if" part of (A) and possibly also omitting (B). This would make no difference in our main result, the characterization of uniformly convex spaces below, since the additional hypotheses used there are sufficient to imply the omitted axioms.

3. Uniform convexity. A normed real linear space $N$ is uniformly convex if for every positive $\epsilon$ there is a positive $\delta$ such that, for $x, y \in N$,

$$
\|x\| \leq 1, \quad\|y\| \leq 1, \text { and }\|x-y\| \geq \epsilon \text { imply }\left\|\frac{1}{2}(x+y)\right\| \leq 1-\delta .
$$

An equivalent condition is that, for sequences $\left\{x_{n}\right\},\left\{y_{n}\right\}$ of vectors in $N$ of norm $\leq 1$, if $\lim _{n \rightarrow \infty}\left\|\frac{1}{2}\left(x_{n}+y_{n}\right)\right\|=1$, then $\lim _{n \rightarrow \infty}\left\|x_{n}-y_{n}\right\|=0$. In the paper [2] in which he introduced the concept of uniform convexity, Clarkson showed that the classical Banach spaces $L_{p}(1<p<\infty)$ are uniformly convex. For $p \geq 2$, the proof is based on the inequality

$$
\|(a+b) / 2\|^{p}+\|(a-b) / 2\|^{p} \leq \frac{1}{2}\|a\|^{p}+\frac{1}{2}\|b\|^{p},
$$

where the norm is the $L_{p}$-norm. When $a$ and $b$ are on the unit sphere, the right side of (4) reduces to 1 , and we obtain Axiom $\left(C^{*}\right)$ for the probability function $\|(a+b) / 2\|^{p}$, a function for which Axioms (A) and (B) are easily seen to hold as well. Thus, Clarkson's inequality (4) implies that the unit sphere of $L_{p}$ is a partial Mielnik space with the above probability function. Note that, when $p=2$, we obtain again the probability function (1) of $\S 1$. For other values of $p$, this partial Mielnik space is not a probability space because $L_{p}$ is not a Hilbert space.

To relate uniform convexity of a space $N$ to partial Mielnik space structures on its unit sphere $S$, we need the following characterization of uniformly convex spaces.

LEMMA. A normed real linear space $N$ is uniformly convex if and only if, for all sequences $\left\{a_{n}\right\},\left\{b_{n}\right\}$ of unit vectors,

$$
\lim _{n \rightarrow \infty}\left\|a_{n}+b_{n}\right\|=2 \text { implies } \lim _{n \rightarrow \infty}\left\|a_{n}-b_{n}\right\|=0 .
$$


Proof. This lemma differs from the remark immediately following the definition of uniform convexity (whose proof is easy and may be found in [3]) only in that $a_{n}$ and $b_{n}$ have length exactly 1 rather than $\leq 1$. Thus, the "only if" part of the lemma is clear. To prove the converse, assume the stated condition and suppose $x_{n}, y_{n}$ are vectors of length $\leq 1$ with $\lim _{n \rightarrow \infty}\left\|x_{n}+y_{n}\right\|$ $=2$. From this and the triangle inequality, we obtain $\lim _{n \rightarrow \infty}\left\|x_{n}\right\|$ $=\lim _{n \rightarrow \infty}\left\|y_{n}\right\|=1$. So we may assume none of the $x$ 's or $y$ 's is 0 , and we may define $a_{n}=x_{n} /\left\|x_{n}\right\|$ and $b_{n}=y_{n} /\left\|y_{n}\right\|$. Now

$$
\left\|x_{n}-a_{n}\right\|=\left\|\left(\left\|x_{n}\right\|-1\right) a_{n}\right\|=1-\left\|x_{n}\right\| \rightarrow 0 \text { as } n \rightarrow \infty,
$$

and similarly $\left\|y_{n}-b_{n}\right\| \rightarrow 0$ as $n \rightarrow \infty$. Then

$$
\left\|a_{n}+b_{n}\right\| \geq\left\|x_{n}+y_{n}\right\|-\left\|x_{n}-a_{n}\right\|-\left\|y_{n}-b_{n}\right\| \rightarrow 2,
$$

and as $\left\|a_{n}+b_{n}\right\| \leq 2$ for all $n$, we have $\lim _{n \rightarrow \infty}\left\|a_{n}+b_{n}\right\|=2$. By hypothesis, this implies $\left\|a_{n}-b_{n}\right\| \rightarrow 0$ and thus

$$
\left\|x_{n}-y_{n}\right\| \leq\left\|x_{n}-a_{n}\right\|+\left\|a_{n}-b_{n}\right\|+\left\|b_{n}-y_{n}\right\| \rightarrow 0 .
$$

Half of the following theorem follows easily from the preceding lemma. We remind the reader that $\mathscr{F}$ was defined in $\S 1$ to be the class of continuous and strictly increasing functions from $[0,2]$ to $[0,1]$. with $f(0)=0, f(2)=1$.

THEOREM. Let $N$ be a normed real linear space, and let $S$ be its unit sphere, $N$ is uniformly convex if and only if, for some $f \in \mathscr{F}$, the probability function $p(a, b)=f(\|a+b\|)$ makes $S$ a partial Mielnik space.

Proof. If. We use the criterion for uniform convexity given by the lemma. Suppose $a_{n}$ and $b_{n}$ are unit vectors such that $\left\|a_{n}+b_{n}\right\| \rightarrow 2$ as $n \rightarrow \infty$. By Axiom $\left(\mathrm{C}^{*}\right)$ for $p$, we have

$$
f\left(\left\|a_{n}+b_{n}\right\|\right)+f\left(\left\|a_{n}-b_{n}\right\|\right) \leq 1,
$$

for it is easy to check, using the definition of $\mathscr{F}$, that the bases of $(S, p)$ are the sets $\{b,-b\}$ for arbitrary $b \in S$. Taking the limit of (5) as $n \rightarrow \infty$ and using the continuity of $f$, we find

$$
f\left(\lim _{n \rightarrow \infty}\left\|a_{n}+b_{n}\right\|\right)+\lim _{n \rightarrow \infty} f\left(\left\|a_{n}-b_{n}\right\|\right) \leq 1 .
$$

But $\lim _{n \rightarrow \infty}\left\|a_{n}+b_{n}\right\|=2$ and $f(2)=1$; since $f$ is nonnegative, we obtain

$$
\lim _{n \rightarrow \infty} f\left(\left\|a_{n}-b_{n}\right\|\right)=0 .
$$

Finally, as $f^{-1}$ is continuous (for $f$ is continuous and strictly increasing), we deduce $\lim _{n \rightarrow \infty}\left\|a_{n}-b_{n}\right\|=0$.

Before proving the converse, we point out that the preceding argument used far less than the full strength of the hypotheses. We did not use Axioms (A) and (B) for $p$, and the appeal to the monotonicity of $f$ at the last step could have been avoided; we only needed that $f(t)=0$ implies $t=0$. (Granting this, we obtain from (6) that no subsequence of $\left\{\left\|a_{n}-b_{n}\right\|\right\}$ can have a 
nonzero limit. Since this sequence lies in the compact set [0,2], it must converge to 0 .) On the other hand, if we are willing to use the full strength of strict monotonicity, then, as is easily seen, we can weaken the continuity assumption to require continuity of $f$ only at 2 .

Only if. We are given a uniformly convex space $N$, and we seek $f \in \mathscr{F}$ such that $p(a, b)=f(\|a+b\|)$ makes the unit sphere $S$ a partial probability space. Regardless of the choice of $f$, Axioms (A) and (B) will hold. (The "only if" part of (A) depends on the strict convexity of $N$, an immediate consequence of uniform convexity.) Thus, the problem is to find $f \in \mathscr{F}$ such that $\left(\mathrm{C}^{*}\right)$ holds, i.e., for all $a, b \in S$,

$$
f(\|a+b\|)+f(\|a-b\|) \leq 1 .
$$

It suffices to find $f$ and $g$ in $\mathscr{F}$ such that, for all $a, b \in S, f(\|a+b\|)$ $+g(\|a-b\|) \leq 1$, for then $\min \{f, g\}$ is in $\mathscr{F}$ and satisfies (7). We select $f(t)=t / 2$ and therefore seek $g \in \mathscr{F}$ such that, for all $a, b \in S, g(\|a-b\|)$ $\leq 1-\frac{1}{2}\|a+b\|$. For any $t \in[0,2]$, define

$$
h(t)=\sup \{\|a+b\|: a, b \in S,\|a-b\| \geq t\} .
$$

We thus require (as $g$ is to be monotone) $g(t) \leq 1-h(t) / 2$ for all $t \in[0,2]$. It is clear that $h$ is monotone nonincreasing and that $h(0)=2$. The fact that $N$ is uniformly convex shows that $h(t)<2$ for all $t \neq 0$. Also, the strict convexity of $N$ implies that $h(2)=0$, for $\|a-b\|$ can equal 2 only when $a=-b$. Finally, the uniform convexity of $N$ implies that $h$ is continuous at 2 . We would like to set $g(t)=1-h(t) / 2$, but it is not clear that this function is continuous or strictly monotone. It does, however, satisfy the hypotheses of the following lemma, so the proof of the Theorem will be complete once the lemma is established.

LemMA. Suppose $g$ maps $[0,2]$ into $[0,1]$, is monotone nondecreasing, takes the value 0 at 0 and nowhere else, maps 2 to 1 , and is continuous at 2 . Then there is $a g_{1} \in \mathscr{F}$ such that $g_{1}(t) \leq g(t)$ for all $t \in[0,2]$.

Proof. Define $g_{1}$ by the Riemann-Stieltjes integral

$$
g_{1}(t)=\int_{u=0}^{t} \frac{t-u}{2-u} d g(u) .
$$

We are thinking of $g$ as describing a distribution of unit mass on the interval $[0,2] ; g(t)=$ mass on $[0, t]$. The jumps of $g$ correspond to discrete mass points. The hypotheses on $g$ say that there is no (discrete) mass at 0 or at 2 and that every nondegenerate interval of the form $[0, t]$ contains some mass. Let us take this mass distribution and smear it, distributing the mass located at $u$ uniformly over the interval $[u, 2]$. The resulting distribution, described by $g_{1}$, no longer has any discrete concentrations of mass because these have been smeared out. (It is essential here that there was no discrete mass at 2.) So $g_{1}$ is continuous. Also, every nondegenerate interval now contains some of the mass originally located in $[0, t]$ for small nonzero $t$. So $g_{1}$ is strictly increasing. $g_{1}(0)=0$ and $g_{1}(2)=1$ are clear. Finally $g_{1}(t)$ is clearly $\leq g(t)$ (since mass 
was moved only to the right). This completes the proof of the Lemma and thus the proof of the Theorem as well.

4. Additional remarks. The comments we made after the proof of the "if" part of our Theorem show that the Theorem remains true if the class $\mathscr{F}$ is replaced by either

$$
\begin{aligned}
\mathscr{F}_{1}=\{f \mid f:[0,2] \rightarrow[0,1], & f \text { strictly increasing, } \\
& \text { continuous at } 2, f(0)=0, \quad f(2)=1\}
\end{aligned}
$$

or

$$
\begin{aligned}
& \mathscr{F}_{2}=\{f \mid f:[0,2] \rightarrow[0,1], f \text { continuous, } \\
& \qquad f(2)=1, \text { and } f(t)=0 \text { iff } t=0\} .
\end{aligned}
$$

Furthermore, the Theorem remains true if Axioms (A) and (B) are omitted from the definition of partial Mielnik spaces. Although this observation permits us to redefine partial Mielnik spaces so as to include examples like the one discussed in the last paragraph of $\$ 2$ (where the "if" part of (A) was false and (B) not immediately clear), the added generality would be irrelevant in our Theorem as both the "if" part of (A) and (B) hold for any $p$ of the assumed form $f(\|a+b\|), f \in \mathscr{F}$ (or $\mathscr{F}_{1}$ or $\mathscr{F}_{2}$ ). Notice, however, that the Theorem remains true if we put the inequality $p(a, b) \geq f(\|a+b\|)$ in place of equality, and then (B) no longer follows.

Another proof of the last lemma in $\$ 3$ was pointed out to us by $B$. Baishanski. One expresses the given $g$ as the sum of a continuous nondecreasing function and an infinite series of nondecreasing step functions each having only finitely many jumps. Since the series converges uniformly, one obtains the required $g_{1}$ by replacing each of these step functions by a continuous piecewise linear minorant having the same value at 2. (Again it is essential that $g$ has no jump at 2.)

We close this paper with a brief and incomplete discussion of conditions under which a partial Mielnik space $(S, p)$ is a subspace of a Mielnik space. We define orthogonality and bases exactly as in probability spaces. It is no longer true, in general, that all bases have the same cardinality. Note, however, that in the spaces $(S, p)$ occurring in the Theorem of $\S 3$, all bases have cardinality 2, for $a$ and $b$ are orthogonal iff $a=-b$. If $a \in S$ and $B \subseteq S$, we define

$$
p(a, B)=p(B, a)=\sum_{b \in B} p(a, b)
$$

and if $C \subseteq S$ also,

$$
p(B, C)=\sum_{b \in B} p(b, C)=\sum_{c \in C} p(B, c)=\sum_{b \in B} \sum_{c \in C} p(b, c) .
$$

Thus, for example, $(S, p)$ is a Mielnik space iff $p(a, B)=1$ for all $a \in S$ and all bases $B$ of $S$. For orthogonal systems (i.e. sets of pairwise orthogonal elements) $B$ and $C$, we write $B \leq C$ to mean that $p(b, C)=1$ for all $b \in B$. Axiom (A) guarantees that $\leq$ is reflexive; transitivity of $\leq$ follows immedi- 
ately from the following condition (D) which holds in some, but by no means all, partial Mielnik spaces:

(D) If $B \leq C$ (where $B$ and $C$ are orthogonal systems) then, for all $a \in S, p(a, B) \leq p(a, C)$.

Proposition 1. If the partial Mielnik space $(S, p)$ is a subspace of a Mielnik space $(\bar{S}, \bar{p})$, then $(S, p)$ satisfies condition $(\mathrm{D})$.

Proof. Suppose $a \in S$ and $B \leq C$ in $S$. As an orthogonal system in $\overline{S,} C$ can be extended to a basis $\bar{C}$ in $\bar{S}$. Since $B \leq C$, we have, for each $b \in B$,

$$
1=p(b, C)=\bar{p}(b, C) \leq \bar{p}(b, \bar{C})=1,
$$

which can hold only if $\bar{p}(b, \bar{C}-C)=0$. Thus, $B \cup(\bar{C}-C)$ is an orthogonal system in $\bar{S}$ and may be extended to a basis $\bar{B}$. Then we have both

$$
1=\bar{p}(a, \bar{C})=p(a, C)+\bar{p}(a, \bar{C}-C)
$$

and

$$
1=\bar{p}(a, \bar{B}) \geq p(a, B)+\bar{p}(a, \bar{C}-C),
$$

so $p(a, B) \leq p(a, C)$, and condition (D) holds.

Condition (D), which we have just shown to be necessary for embeddability into a Mielnik space, in some circumstances is also sufficient. We prove here just one result of this sort; the additional hypothesis about the bases is unnecessarily restrictive, but it holds in the spaces $(S, p)$ considered in $\S 3$.

Proposition 2. If condition (D) holds in a partial Mielnik space $(S, p)$ all of whose bases have the same finite cardinality $n$, then $(S, p)$ is a subspace of a Mielnik space (of dimension $2 n$ ).

Proof. We show first that, under these hypotheses, the relation $\leq$, restricted to bases, is symmetric and, therefore, an equivalence relation. For, if $B \leq C$ are bases, then, as $B$ has $n$ elements, $n=p(B, C)=\sum_{c \in C} p(c, B)$. This sum has $n$ terms, each $\leq 1$ by Axiom $\left(C^{*}\right)$; for the sum to be $n$, all terms must be 1 , so $C \leq B$.

For each equivalence class $[B]=\{C \mid C$ a basis, $C \leq B\}$ of bases, add $n$ new elements $[B]_{i}(1 \leq i \leq n)$ to $S$. On the resulting set $\overline{\bar{S}}$, define $\bar{p}$ by

$$
\begin{aligned}
& \bar{p}(a, b)=p(a, b) \text { if } a, b \in S, \\
& \bar{p}\left(a,[B]_{i}\right)=\bar{p}\left([B]_{i}, a\right)=n^{-1}(1-p(a, B)) \text { for } a \in S, 1 \leq i \leq n, \\
& \bar{p}\left([B]_{i},[C]_{j}\right)=n^{-2} p(B, C) \text { if }[B] \neq[C], \\
& \bar{p}\left([B]_{i},[B]_{j}\right)=0 \text { if } i \neq j, \\
& \bar{p}\left([B]_{i},[B]_{i}\right)=1 .
\end{aligned}
$$

It is entirely straightforward (though tedious) to verify that $(\bar{S}, \bar{p})$ is a probability space. Its bases have the form $B \cup\left\{[B]_{i} \mid 1 \leq i \leq n\right\}$ where $B$ is a basis for $S$, so $\bar{S}$ has dimension $2 n$.

\section{REFERENCES}

1. G. Birkhoff and J. von Neumann, The logic of quantum mechanics, Ann. of Math. (2) 37 (1936), 823-843. 
2. J. A. Clarkson, Uniformly convex spaces, Trans. Amer. Math. Soc. 40 (1936), 396-414.

3. G. Köthe, Topologische Lineare Räume. I, Die Grundlehren der math. Wissenschaften, Band 107, Springer-Verlag, Berlin, 1966; English transl., Die Grundlehren der math. Wissenschaften, Band 159, Springer-Verlag, New York, 1969. MR 33 \#3069; 40 \#1750.

4. B. Mielnik, Geometry of quantum states, Comm. Math. Phys. 9 (1968), 55-80. MR 37 \#156.

5. C. V. Stanojević, Mielnik's probability manifolds and inner product spaces, Bull. Acad. Polon. Sci. Sér. Sci. Math. Astronom. Phys. 18 (1970), 531-532. MR 42 \#259.

6. - Mielnik's probability spaces and characterization of inner product spaces, Trans. Amer. Math. Soc. 183 (1973), 441-448.

Department of Mathematics, University of Michigan, ANN Arbor, Michigan 48104

Department of Mathematics, University of Missouri, Rolla, Missouri 65401 